\title{
A Modified Method for Bone Regeneration using Gelatin Sponge with Bone Graft in treating an Osseous Defect after Root Resection
}

\author{
${ }^{1}$ Sathyanarayana Sunil, ${ }^{2}$ Harsha M Babu
}

\begin{abstract}
Introduction: Modern advances in all phases of dentistry have provided the opportunity for patients to maintain a functional dentition for longer periods of time. The disciplines of endodontics, periodontics, and prosthodontics fuse when the molars that have furcation involvements are treated by hemisection or root resection. Preserving the socket in these cases after removing the root or tooth segment and regenerating bone around the remaining portion of the root is an important part of the treatment that determines the prognosis of the tooth.
\end{abstract}

Case report: To enhance bone regeneration, a modified approach was used while bone grafting, where gelatin sponge was placed along with the synthetic hydroxyapatite and $\beta$-tricalcium phosphate in the osseous defect of the lower right first molar tooth following distal root resection. In this case report, the tooth after root resection has shown profound improvement in periodontal health, and the socket was preserved satisfactorily.

Conclusion: The simple modification showed an uneventful healing and excellent periodontal health clinically and good bone fill contributing to socket preservation as seen radiographically. The tooth has continued to serve well as found in the sixth month follow-up.

Clinical significance: The future prospect of using bone graft along with an agent that holds the clot and stabilizes it (gelatin sponge) during healing could serve as a useful treatment procedure, as this combination might act together producing synergistic effects.

Keywords: Gelatin sponge, Root resection, Socket preservation.

How to cite this article: Sunil S, Babu HM. A Modified Method for Bone Regeneration using Gelatin Sponge with Bone Graft in treating an Osseous Defect after Root Resection. J Health Sci Res 2017;8(2):80-83.

Source of support: Nil

Conflict of interest: None

\section{INTRODUCTION}

Periodontal disease involving severe bone loss in the furcation area and around a root in multirooted teeth

\footnotetext{
${ }^{1}$ Associate Professor, ${ }^{2}$ Reader

1,2Department of Periodontics, Dayananda Sagar College of Dental Sciences, Bengaluru, Karnataka, India

Corresponding Author: Harsha M Babu, Reader, Department of Periodontics, Dayananda Sagar College of Dental Sciences Bengaluru, Karnataka, India, Phone: +919845735007, e-mail: harshamb@yahoo.com
}

poses a great challenge for salvaging teeth and restoring function. Tooth resection procedures in which one or more roots with or without associated coronal tooth structures are removed, are viable, and are accepted ways to manage teeth with compromised osseous, radicular, and restorative presentations. ${ }^{1}$

Preserving the socket in these cases after removing the root or tooth segment and regenerating bone around the remaining portion of the root is an important part of the treatment that determines the prognosis of the tooth. To fulfill this objective, a modified technique was used to enhance bone regeneration, where gelatin sponge was placed along with the synthetic hydroxyapatite and $\beta$-tricalcium phosphate in the osseous defect.

\section{CASE REPORT}

A 37-year-old male patient reported with a chief complaint of bleeding gums since 2 years. He was diagnosed having chronic generalized periodontitis. Following scaling and root planing, flap surgeries were performed in all quadrants and the patient was placed on maintenance therapy. The patient practiced good oral hygiene and showed good compliance, but continuing attachment loss was recorded clinically in the lower right first molar. In about 6 months, the probing pocket depth recorded was $>10 \mathrm{~mm}$ around the distobuccal and distolingual regions. The probing depth was normal around the mesial aspects of the tooth. The overlying gingiva was inflamed, Miller's grade II mobility of the tooth was recorded, and intraoral radiograph showed bone loss in the furcation region, which extended beyond the apex of the distal root. Owing to the presence of adequate bone support around the mesial root and patient's intense desire to retain the tooth, endodontic treatment for the tooth and resection of the distal root were planned.

\section{TREATMENT}

Endodontic treatment of the tooth was done, and since resection of distal root was planned, after biomechanical preparation of all canals, only the mesial root canals were obturated (Fig. 1). After adequate local anesthesia using $2 \%$ lidocaine (1:1,00,000 adrenaline), a full-thickness envelop flap was elevated (Fig. 2). Resection of the distal 


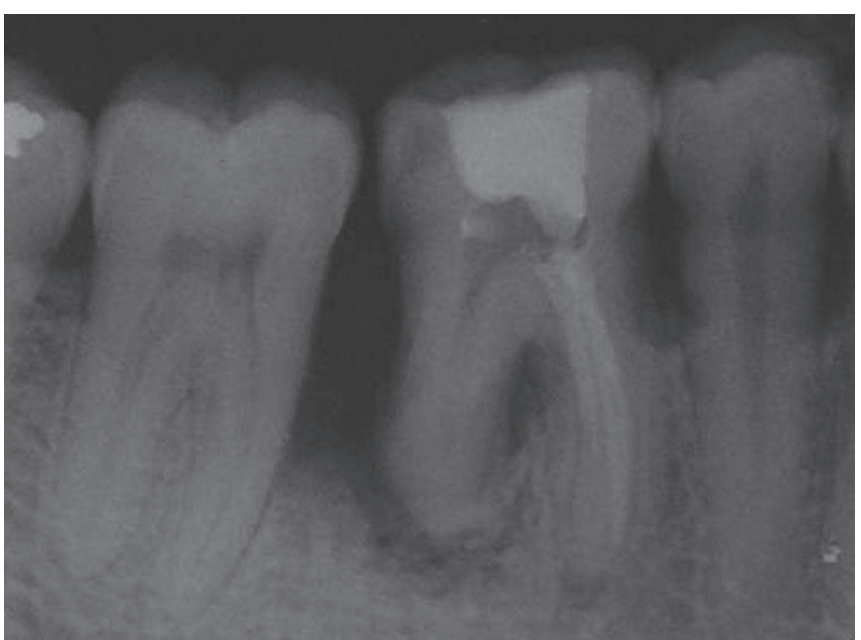

Fig. 1: Preoperative radiograph showing extensive bone loss around the distal root and obturated mesial root canals

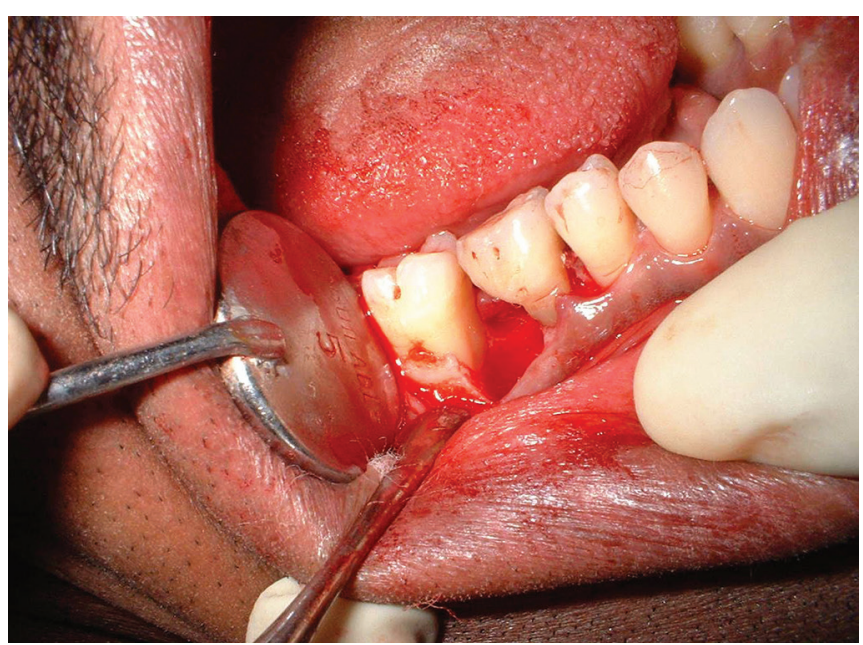

Fig. 3: Surgical site after resection of the distal root

root was carried out by sectioning the root at the furcation area and the ridges were smoothened to remove any plaque-retentive areas (Fig. 3). The resected distal portion of the root showed two separate buccal and lingual roots (Fig. 4). Osteoplasty was done to obtain a proper flap adaptation. Synthetic hydroxyapatite plus $\beta$-tricalcium phosphate bone graft mixed with small pieces of gelatin sponge, which could act as a scaffold for bone formation, was filled to the height of the remaining socket walls and sutured (Fig. 5). Care was taken not to expose the gelatin sponge to the oral environment by getting a complete closure of the flap. Coronoplasty was performed on the distal portion to reduce the occlusal forces on the unsupported part of crown. Sutures were removed at the end of 1 week and the healing was uneventful. By the end of the 1st month, the tooth was clinically stable. The radiograph showed radiopaque area simulating bone fill at the distal root region (Fig. 6). At 6-months follow-up visit, the periodontal tissues were clinically (Fig. 7) and radiographically evaluated, which showed excellent bone

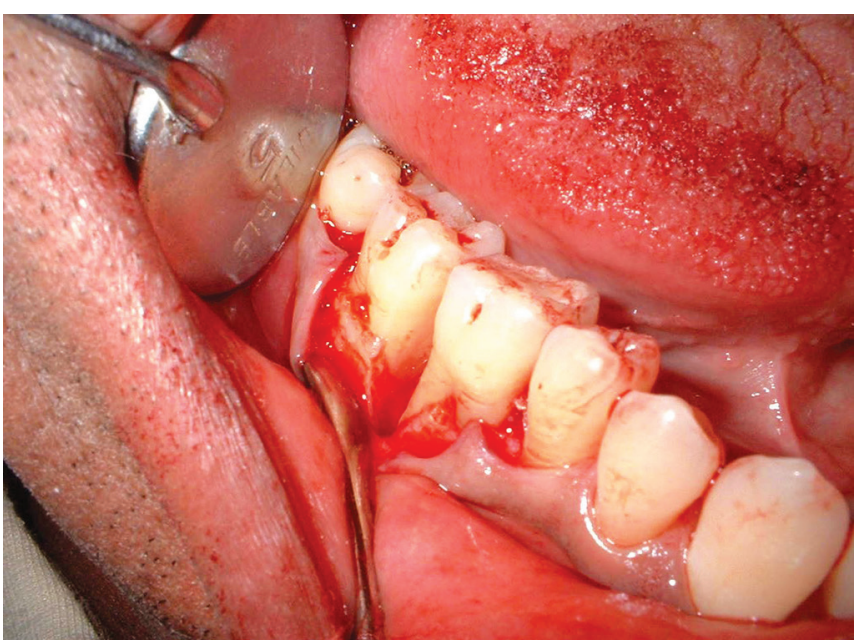

Fig. 2: Full-thickness envelop flap elevated showing osseous defect

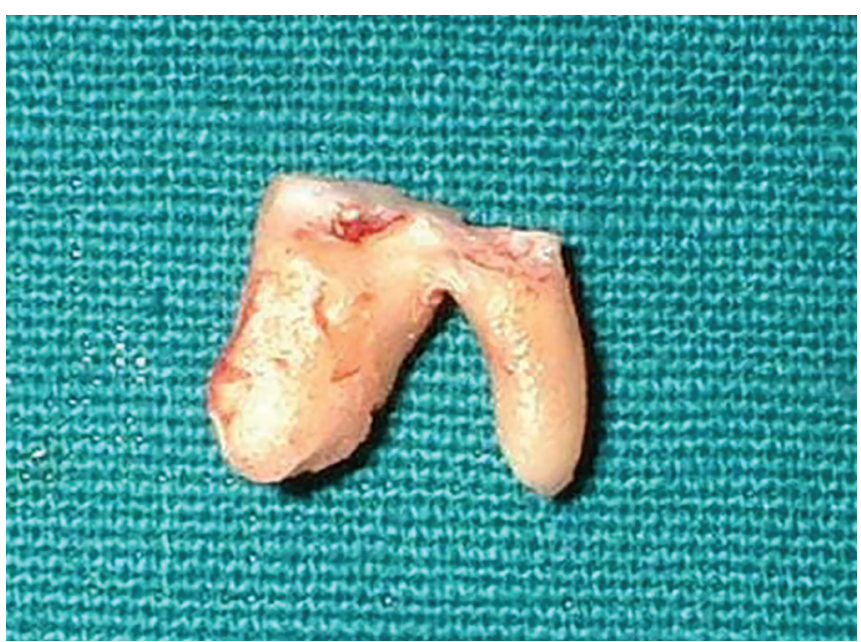

Fig. 4: Distal root showing separate buccal and lingual roots

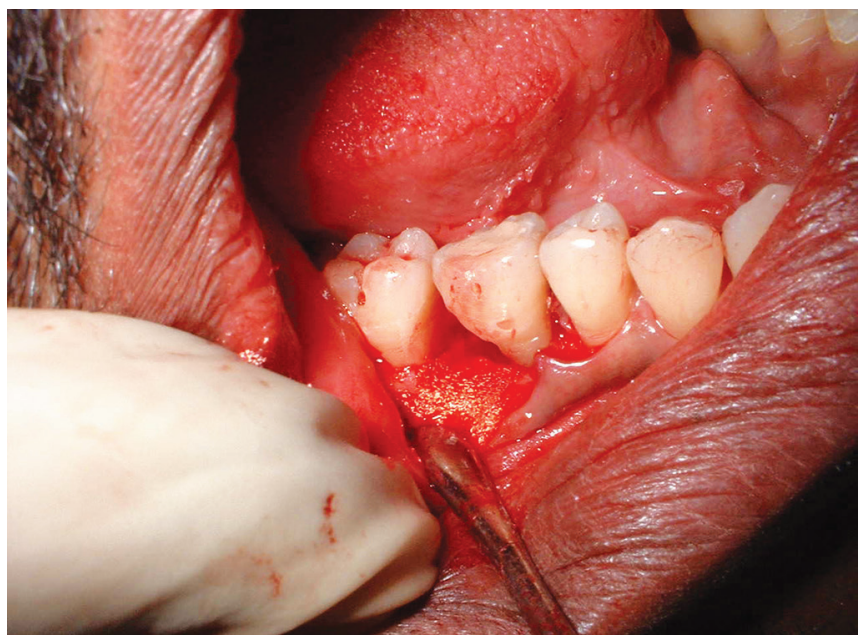

Fig. 5: Bone graft and gelatin sponge placed in the osseous defect

fill in the socket (Fig. 8), indicating acceptance of gelatin and bone graft material. The initial probing pocket depth, which was more than $10 \mathrm{~mm}$ around the distobuccal and distolingual regions, now showed $2 \mathrm{~mm}$ probing depth. 


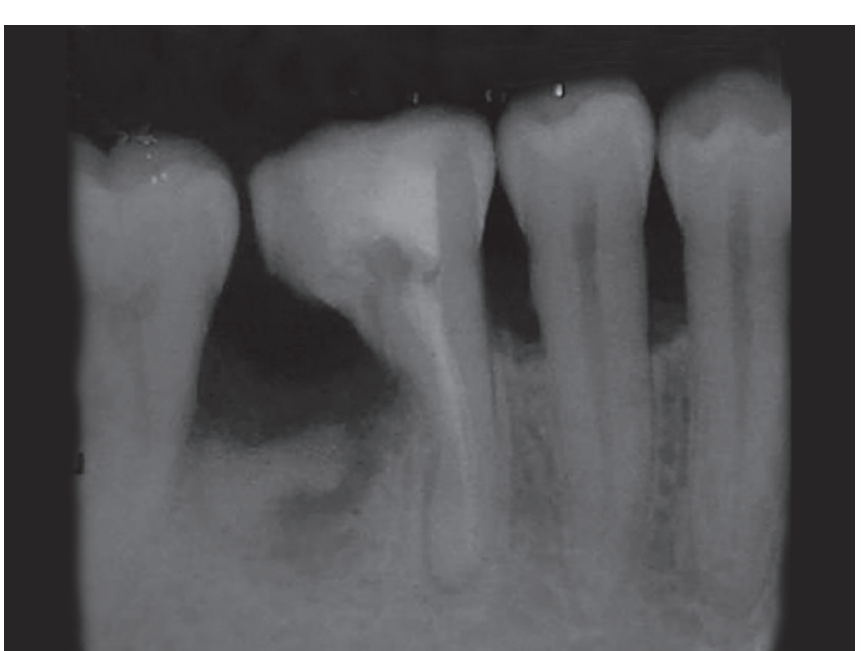

Fig. 6: One-month follow-up radiograph showing good bone density at the distal root region

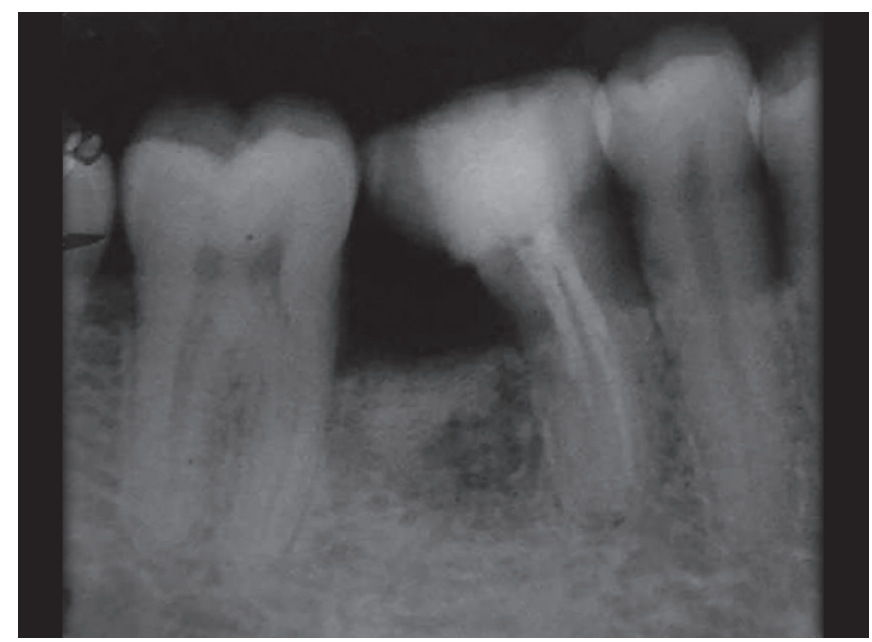

Fig. 8: Six-months follow-up radiograph showing good bone density at the distal root region

The tooth which was Miller's grade II mobile at the time of surgery had become completely firm at 6 months, indicating excellent recovery and pointing the etiology of periodontal destruction toward the bifurcated distal root.

\section{DISCUSSION}

Root resection therapy is a treatment option for molars with periodontal, endodontic, or prosthetic problems. Since root resection is technique sensitive and complex, proper case selection is essential. ${ }^{2}$ The prognosis of root resection is well documented in previous studies. Carnevale et $\mathrm{al}^{3}$ demonstrated that the survival rate of resected teeth, during the 10-year period of observation, reached $93 \%$. Langer et $\mathrm{al}^{4}$ reported a $62 \%$ success rate for the resected molars over a 10 -year period; $26 \%$ of failures were due to periodontal problems. Bühler ${ }^{5}$ reported that $68.9 \%$ of resected molars survived, and $44 \%$ of failures were related to periodontal problems. Blomlöf et $\mathrm{al}^{6}$ showed similar results, in that $32 \%$ of molars failed over a

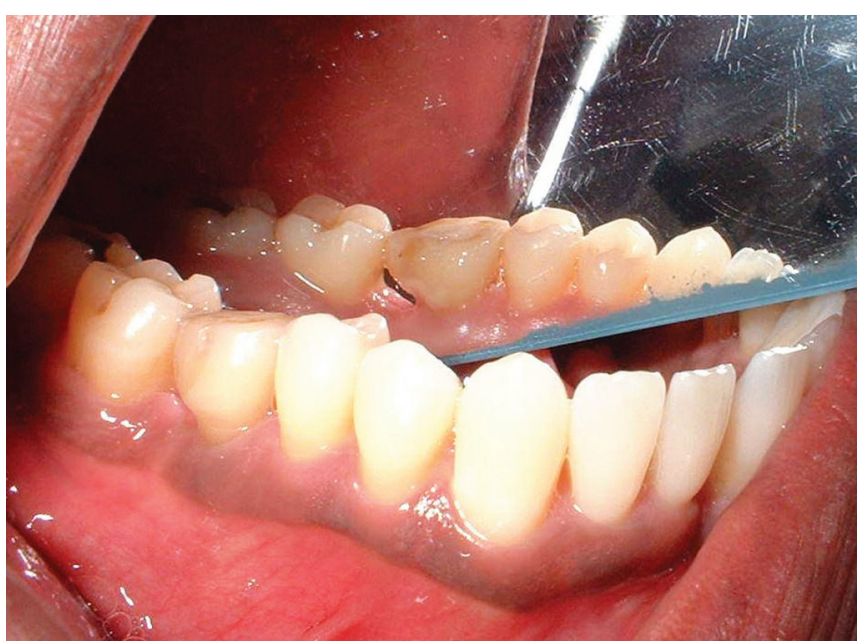

Fig. 7: Six-months postoperative picture showing clinically healthy periodontal tissues

10-year period; $80 \%$ of the failed molars were lost because of periodontal or endodontic-periodontal lesions. The survival rate of resected molars might be influenced by a variety of factors. Park et $\mathrm{al}^{7}$ categorized these factors into four groups: Resection-, patient-, tooth-, and site-related factors. They concluded that root resection to treat periodontal problems showed a better prognosis than root resection performed for nonperiodontal purposes. To achieve good results in periodontally diseased molars, more than $50 \%$ bone support of the remaining roots at the time of the root resection is an important factor. In the present case report, root resection was carried out due to periodontal reasons, and the bone support on the remaining mesial root was greater than $50 \%$. The gelatin sponge used as a local hemostatic agent resorbs in 4- to 6-week time when left inside, and this allows time for the organization of the clot. The basis for selection of gelatin sponge with bone graft was that it swells up when it comes in contact with blood and holds the clot, and might also act as a scaffold allowing vessel in-growth. It also maintains the space until it resorbs. It can absorb 40 to 50 times its own weight of blood and might bring in more growth factors to the defect site leading to better regeneration outcomes. ${ }^{8}$ Rohanizadeh et $\mathrm{al}^{9}$ investigated the ability of gelatin scaffolds to be used as a support for osteoblast proliferation and differentiation and reported that tested scaffolds demonstrated the ability to support osteoblast activities and allowed cell proliferation and cell migration into the sponge porosities. Another reason for using gelatin sponge was its easy availability and economy.

The clinical results of our case suggest that gelatin fragments might function as a carrier of natural growth factors, and act as a filler to prevent the growth of soft tissue into the periodontal defect, which is in concurrence with a case series reported by Kabashima et $\mathrm{al}_{1}^{10}$ who concluded that application of an autologous blood clot combined with 
gelatin was the simplest and safest procedure for induction of tissue healing and regeneration in periodontal defects. Vandana et $\mathrm{al}^{11}$ reported successful clinical attachment gain, decreased probing pocket depth, and satisfactory defect fill of intrabony defect with the use of Abgel ${ }^{\circledR \odot \mathrm{TM}}$ (gelatin sponge - scaffold) over 1-year follow-up period.

This case evidenced one such better outcome, which should make way for future long-term studies with large study samples to give gelatin sponge a more regular place in regenerative procedures.

\section{CONCLUSION}

The prognosis of root resection depends on the case selection, adequately performed endodontic treatment, and the bone support around the remaining root. The future prospect of using bone graft along with an agent that holds the clot and stabilizes it (gelatin sponge) during healing could serve as a useful treatment procedure and should not be overlooked, as this economical combination might act together producing synergistic effects. Further studies can provide evidence for the effective utility.

\section{REFERENCES}

1. Gutmann, JL. Perforative repair, resection procedures and periodontal management. In: Gutmann JL, editor. Gutmann's surgical endodontics. 1st ed. Chennai: All India Publishers \& Distributors; 1999. p. 409-448.
2. DeSanctis M, Murphy KG. The role of resective periodontal surgery in the treatment of furcation defects. Periodontol 20002000 Feb;22:154-168.

3. Carnevale G, Pontoriero R, di Febo G. Long-term effects of root-resective therapy in furcation-involved molars. A 10-year longitudinal study. J Clin Periodontol 1998 Mar;25(3):209-214.

4. Langer B, Stein SD, Wagenberg B. An evaluation of root resections. A ten-year study. J Periodontol 1981 Dec;52(12): 719-722.

5. Bühler H. Evaluation of root-resected teeth. Results after 10 years. J Periodontol 1988 Dec;59(12):805-810.

6. Blomlöf L, Jansson L, Appelgren R, Ehnevid H, Lindskog S. Prognosis and mortality of root-resected molars. Int J Periodontics Restorative Dent 1997 Apr;17(2):191-201.

7. Park SY, Shin SY, Yang SM, Kye SB. Factors influencing the outcome of root-resection therapy in molars: a 10-year retrospective study. J Periodontol 2009 Jan;80(1):32-40.

8. Johnson, BS. Procoagulant, anticoagulant and thrombolytic drugs. In: Yagiela JA, editor. Pharmacology and therapeutics for dentistry. 6th ed. St. Louis (MO): Elsevier; 2011. p. 487-510.

9. Rohanizadeh R, Swain MV, Mason RS. Gelatin sponges (Gelfoam) as a scaffold for osteoblasts. J Mater Sci Mater Med 2008 Mar;19(3):1173-1182.

10. Kabashima H, Sakai T, Mizobe K, Nakamuta H, Kurita K, Terada Y. The usefulness of an autologous blood clot combined with gelatin for regeneration of periodontal tissue. J Oral Sci 2013;55(4):363-366.

11. Vandana KL, Desai R, Dalvi PJ. Autologous stem cell application in periodontal regeneration technique (SAI-PRT) using PDLSCs directly from an extracted tooth-an insight. Int J Stem Cells 2015 Nov;8(2):235-237. 